withdrawals and loss to follow-up. This is important because loss to follow-up in a small trial may lead to bias. Although the authors examine the issue of bias due to loss to follow-up, by providing comparison material between the group of all patients and the group with complete ratings, the reader also needs detailed information on those lost to follow-up. For example, in Table 1, the difference between the CST and generic groups in mean number of admissions and mean number of prehospital days increases considerably from the group of "all patients" to the group of "rated patients" (from 0.6 to 2.2, and from 0 to 23 respectively). This could indicate that loss to follow-up had differential effects on the distribution of important predictors of outcome in the two groups. Another bias might have been introduced by the fact that baseline assessment apparently took place after randomisation, with the possibility of influencing initial expectations of both patients and raters. For example, at baseline there are significant differences between the two groups on the GAS (mean difference 4.9; 95\% CI 4.0-5.9) and the related PSE measure (mean difference $4.7 ; 95 \%$ CI $3.4-6$ ). This demonstrates better functioning in CST patients at baseline, a difference that disappeared at subsequent measure points.

Time in hospital. Time spent in hospital, arguably the most important outcome measure of the trial, is usually skewed. In reporting the results on total time spent in hospital, the authors state that the mean time in hospital was $\mathbf{4 6}$ days for the CST and 44 days for the generic group. While this would indicate a similar length of stay, they also report that median length in the CST group was 21 days, but 12 days in the generic group. This would suggest that the CST group had longer admissions, which may be related to the fact that patients in the generic group were more often living in hostel care. This point needs to be clarified; if time in hospital is evidently skewed, then the comparison of mean values as presented in Table 4 is invalidated, and the authors should use the appropriate non-parametric test. The fact that for both groups the median values are much lower than the mean values does indicate that the data are positively skewed. It is therefore important that the reader be provided with the appropriate statistical information as to whether the intervention groups differed beyond chance expectation with respect to this outcome measure.

We agree with Muijen $e t$ al that the issue examined is an important one, and that one should interpret the findings cautiously. However, we feel that their assumption that the negative findings are informative is not entirely justified. Apart from the possibility of a high type II error rate, the results are difficult to interpret because of unsatisfactory adjustment for confounding and the possibility of bias.

Frison, L. \& Pocock, S. J. (1992) Repeated measures in clinical trials: analysis using mean summary statistics and its implications for design. Statistics in Medicine, 11, 1685-1704.

POCOCK, S. J., Hughes, M. D. \& LeE, R. J. (1987a) Statistical problems in the reporting of clinical trials. A survey of three medical journals. New England Journal of Medicine, 317, 426432.

—, Geller, N. \& Tsutis, A. (1987b) The analysis of multiple endpoints in clinical trials. Biometrics, 43, 487-498.

JIM VAN OS

KWAME MCKENZIE

KarYNa GilvarRY

TOM FAHY

Institute of Psychiatry

De Crespigny Park

London SES $8 A F$

\section{Impact of new long-stay patients}

SIR: Lelliott (BJP, August 1994, 165, 160-169 and 170-178) raises the issue of the impact of new long-stay patients (NLS) on acute psychiatric services, who occupied an average of $7 \%$ of acute beds nationally and over $10 \%$ in some services. Our own audit of prolonged admissions to acute wards in Nottingham with onset in 1990 revealed 33 patients with stays of 180 days or more, occupying 8720 bed days. Nottingham has 145 acute beds (including 12 forensic/ITU) for a population of 620000 ( 23 per 100000$)$. There are integrated community mental health services, described in part by Tyrer (1989), and a welldeveloped rehabilitation and community care service.

The patients with prolonged stays accounted for $17 \%$ of available occupancy, and as overall occupancy was then $76 \%, 22 \%$ of actual occupancy. Only two of the NLS patients remained on acute wards for more than one year (maximum stay 421 days). If patients with acute admissions of 90 days or more (Lelliott argues stays of greater than three months are "inhumane") are included, the bed use by the long-stay group rises to $26 \%$ of available, or $33 \%$ of occupied bed days in 1990 . The psychiatric and sociodemographic characteristics of the group appeared similar to that of Lelliott's cohort.

One conclusion that could be drawn from these data is that as services become more community 
orientated and prevent many acute admissions, those patients who do require admission are more seriously ill and often more disturbed. This, combined with the lowering of the denominator of bed numbers, means that this ground will have an increasing impact on acute ward occupancy. This is supported by Dean (1993) who reported two NLS patients blocking a third of the beds in their home treatment service (23 beds per 100000 population) with stays in excess of three years. We felt that many of the prolonged in-patient stays in Nottingham could have been reduced by use of alternative existing services. Acute care was, however, needed for prolonged periods to deal with treatment resistant conditions and to contain high levels of risk. We would agree with Lelliott that an urgent assessment of facilities and management strategies is needed to avoid blocking acute beds with patients who could be better managed by alternative services. This is particularly pertinent in Nottingham as acute occupancy has increased from $75 \%$ to $93 \%$ in the last four years.

Dean, C. (1993) The development of a local service in Birmingham. In Community Mental Health Care: International Perspectives on Making it Happen (eds C. Dean \& H. Freeman) pp. 76-90. London: Gaskell.

TYRer, P., TURner, R. \& Johnson, A. L. (1989) Integrated hospital and community psychiatric services and use of inpatient beds. British Medical Journal, 299, 298-300.

STEFFAN DAVIES SimON PAYNe

Mapperley Hospital

Porchester Road

Nottingham NG7 4GL

\section{The definition of anorexia nervosa}

SIR: The editorial of Drs Parry-Jones (BJP, September 1994, 165, 287-292) demonstrated that whereas the historical syndrome of bulimia bears little consistent resemblance to modern bulimia nervosa, historical data derived from a sizable group of non-fat phobic anorexic patients provides "substantial evidence of continuities" with its modern version, as defined in DSM-III-R or ICD-10. They cautioned against the rigid application of the diagnostic criterion of fat phobia, which has been accorded paramount importance in contemporary nosology.

For several years I have argued for the same thesis - that the patterning of anorectic complaints and the biomedical process of diagnosing anorexia nervosa are both time- and context-dependent. Unlike historical data, which are finite and often incomplete, cross-cultural evidence grows that we may be seeing in non-Western societies a parallel evolution of eating disorder that had historically taken place in the West. A substantial number of non-fat phobic patients fail to fulfil the current diagnostic criteria for anorexia nervosa (Lee et al, 1993).

There is thus an exciting convergence of longitudinal historical and horizontal cross-cultural data. The ideas of Drs Parry-Jones are supported by an increasing number of Western experiments. Yager \& Davis (1993) wrote that "many chronic patients seem far more able to acknowledge that they are too thin", and "we are also very aware of how clinically difficult it can sometimes be to elicit a fat phobia in patients who have evolved a different type of attribution regarding why they are not eating". By conceding that "the same sort of phenomenological heterogeneity occurs in Western anorexia nervosa patients as well", they testified to the intracultural variability of anorexia nervosa in contemporary North America. Steiger (1993) similarly stated that "anyone who works with large numbers of anorexia nervosa sufferers knows that this disorder is not uniformly about a desire to be thin. Rather, the apparent pursuit of thinness or weight phobia seen in anorexia is often explicable in terms idiosyncratic to each case."

Palmer (1993) criticised the central role given to weight concern in the modern diagnosis of anorexia nervosa, as this may have led to "a premature closure" of the inquiry into the nature of the eating disorders in general. Halmi (1994) reiterated that "psychiatric diagnostic categories are constrained by history as well as biology", and even proposed that "the psychobiological vulnerability factors that induced the development of irreversible starvation in medieval saints are similar to those inciting the emergence of anorexia nervosa and bulimia in twentieth-century young women".

The DSM-IV criterion that all anorectic subjects must insist on feeling fat, no matter how emaciated they are, may represent not so much their lived experience as the particular viewpoint of empowered nosologists, and may hamper research work. The bias of any classificatory system is not nearly so bad as the illusion that it is unbiased.

Halma, K. (1994) Princess Margaret of Hungary, 1242-1271. American Journal of Psychiatry, 151, 1216.

LEE, S., Ho, T. P. \& HSU, L. K. G. (1993) Fat phobic and non-fat phobic anorexia nervosa - a comparative study of 70 Chinese patients in Hong Kong. Psychological Medicine, 23, 999-1017. 